Revue des patrimoines

$17 \mid 2011$

Les patrimoines de l'enseignement supérieur

\title{
Les vitraux de l'École de Pharmacie de Paris
}

\section{Françoise Gatouillat}

\section{OpenEdition}

Journals

Édition électronique

URL : http://journals.openedition.org/insitu/946

DOI : 10.4000/insitu.946

ISSN : 1630-7305

\section{Éditeur}

Ministère de la Culture

\section{Référence électronique}

Françoise Gatouillat, « Les vitraux de l'École de Pharmacie de Paris », In Situ [En ligne], 17 | 2011, mis en ligne le 01 décembre 2011, consulté le 10 décembre 2020. URL : http://journals.openedition.org/ insitu/946 ; DOI : https://doi.org/10.4000/insitu.946

Ce document a été généré automatiquement le 10 décembre 2020.

\section{(c) $(1) \&$}

In Situ Revues des patrimoines est mis à disposition selon les termes de la licence Creative Commons Attribution - Pas d'Utilisation Commerciale - Pas de Modification 4.0 International. 


\title{
Les vitraux de l'École de Pharmacie de Paris
}

\author{
Françoise Gatouillat
}

1 Après deux siècles de désaffection, le nouvel élan que connaît la peinture sur verre à partir du règne de Louis-Philippe bénéficie aussi bien aux lieux de culte qu'aux demeures et aux monuments publics. Des années 1840 jusqu'à l'entre-deux guerres, les commandes destinées à l'architecture civile représentent du reste une part non négligeable du chiffre d'affaires des ateliers, certains d'entre eux s'en faisant même une spécialité. L'importance de cette production, méconnue parce qu'une mince partie a survécu à sa place d'origine, est largement attestée par des cartons ou des esquisses, ainsi que par des textes critiques ${ }^{1}$ . Cependant, sur les grands chantiers parisiens de la Troisième république ${ }^{2}$, la peinture et la sculpture jouent seules un rôle majeur. Les verrières réalisées dans les années 1880 pour l'École de Pharmacie, une des rares commandes passées par l'État au XIXe sièclee, illustrent donc une démarche plutôt originale. Aucune des monographies consacrées à l'établissement n'a du reste omis de signaler ce volet de son décor monumental ${ }^{4}$.

\section{La première commande}

2 La construction de la nouvelle École de Pharmacie entre l'avenue de l'observatoire, la rue Michelet et la rue d'Assas, sur un terrain qu'occupait sous l'Ancien Régime la chartreuse de Paris, répond à la nécessité de redéployer l'institution, de longue date trop à l'étroit dans les locaux de la rue de l'Arbalète qui lui avaient été affectés en 1803. Envisagé avant 1870 et financé fin 1875, le chantier débute en avril 1877 sous la direction de Charles-Jean Laisné (1819-1891), architecte diocésain, restaurateur de nombreux monuments ${ }^{5}$. Les bâtiments, aux plans simples et fonctionnels, comprennent un grand corps central et deux ailes en retour sur une large cour bordée d'un péristyle, ouverte sur l'avenue de l'Observatoire ${ }^{6}$. Une vaste galerie occupe la partie médiane, vestibule d'honneur qui donne accès à la salle des Actes, au secrétariat, aux laboratoires, à des salles de lecture et à deux amphithéâtres situés en arrière du corps principal, sur le jardin botanique dont les 
serres se dressent le long de la rue d'Assas. De part et d'autre de l'entrée du vestibule, deux escaliers monumentaux desservent l'étage qui abrite la bibliothèque, le musée de matière médicale ainsi que les collections de zoologie, de physique et de botanique.

Dès l'achèvement du gros œuvre, fin 1881, Charles Laisné se préoccupe du décor intérieur de l'édifice. En janvier 1883, il soumet au ministre de l'Instruction publique et des BeauxArts un rapport sur "la nécessité de décorer les murs du grand vestibule par des peintures d'histoire, et la fenêtre qui l'éclaire par des vitraux en grisaille qui, par leur douce coloration, formeraient un ensemble harmonieux ${ }^{7}$ ». Le peintre Albert Besnard (1849-1934), Grand Prix de Rome en 1874, déjà sollicité directement par l'architecte en mars 1882 pour réaliser les peintures murales, reçoit aussitôt ses premières commandes ${ }^{8}$ (fig. $\mathbf{n}^{\circ} \mathbf{1}$ ). Les décisions nécessaires à la mise en œuvre des vitraux tardant un peu, en avril 1883, l'architecte réitère sa demande en y joignant un devis ${ }^{9}:$ " Pour obtenir l'effet désirable, j'aurais l'honneur de vous prier de confier l'exécution des grisailles [de la baie $\mathrm{du}$ fond du vestibule] à $\mathrm{M}$. Lavergne, dont le style sévère se prêterait à la simplicité des compositions projetées. Les fenêtres des deux grands escaliers pourraient être décorées de vitraux semblables, qui rappelleraient par les sujets qui y seraient traités les faits principaux se rattachant à l'art de préparer les médicaments. M. Hirsch, dont les travaux récents exécutés soit au Trocadéro ${ }^{10}$, soit dans d'autres édifices, ont fait apprécier le mérite, pourrait être chargé de la décoration des escaliers ». La dépense totale, estimée à $22000 \mathrm{~F}$, comprend les $16 \mathrm{~m}^{2}$ de la grande fenêtre du vestibule et les surfaces inégales des quatre fenêtres des escaliers, soit $5,75 \mathrm{~m}^{2}$ pour chaque baie du côté nord et $9,20 \mathrm{~m}^{2}$ pour chacune de celles du sud. Sur cette somme, il est convenu que la direction des Beaux-Arts participe à hauteur de $5500 \mathrm{~F}$ pour les cartons. S'il est d'emblée décidé d'ajourner le projet concernant la verrière du fond du hall, celui d'orner les escaliers est approuvé le 14 juin 1883 et, conformément au vœu de l'architecte, Émile Hirsch se voit confier la réalisation des quatre verrières. Le peintre verrier soumet aussitôt ses esquisses à l'examen d'une commission réunie spécialement par la direction des Beaux-Arts ${ }^{11}$. 
Figure 1

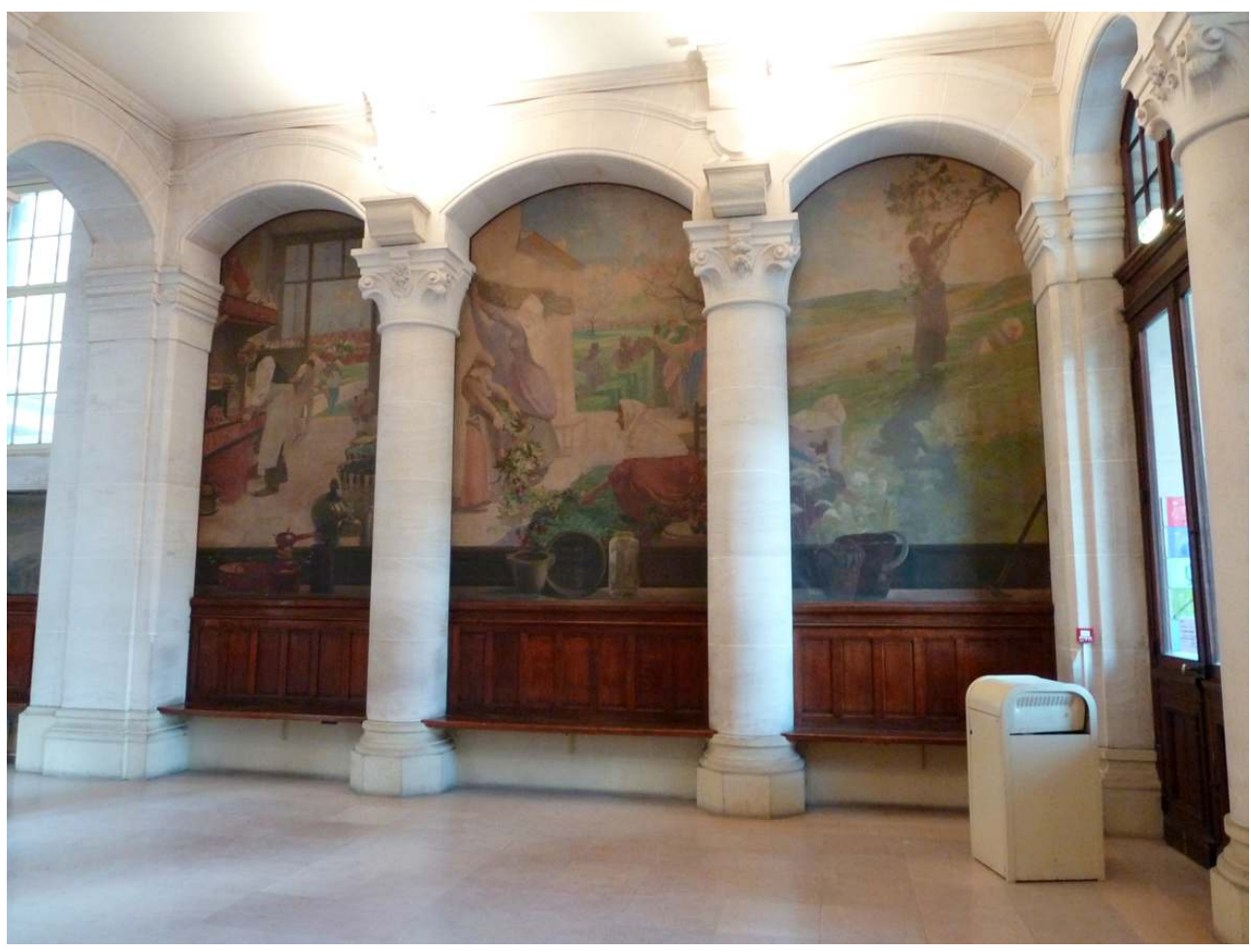

Paris, École de Pharmacie, le vestibule d'honneur décoré par Albert Besnard.

Phot. Françoise Gatouillat.

\section{Un peintre devenu peintre verrier}

4 Lorsqu'il reçoit commande des vitraux de l'École de Pharmacie, Émile Hirsch a déjà honoré de multiples contrats, entre autres pour des églises parisiennes telles que SaintSéverin, Saint-Ferdinand-des-Ternes, Saint-Michel-des-Batignolles ou Saint-Philippe-duRoule (fig. $\left.\mathbf{n}^{\circ} \mathbf{2}\right)$. 


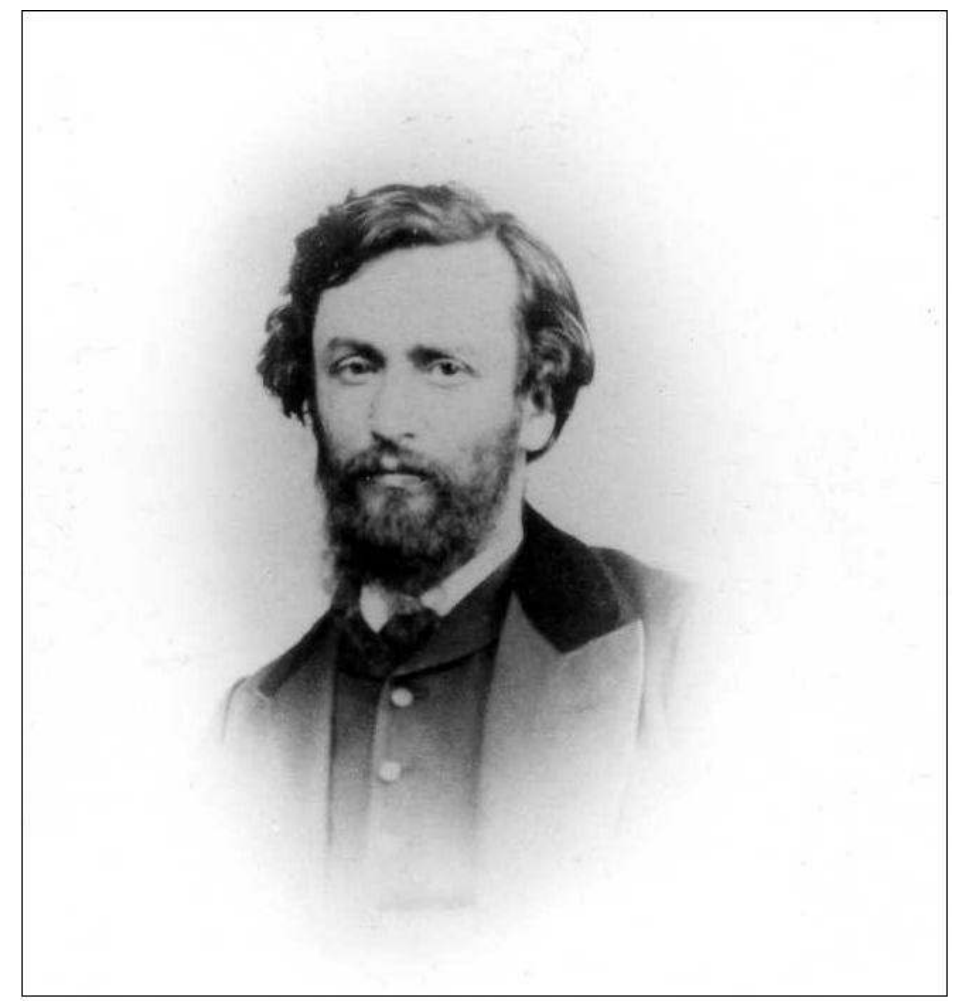

Portrait d'Émile Hirsch, vers 1875.

Collection particulière.

Paul Hartmann, à qui l'on doit l'essentiel de sa biographie, a dénombré près d'un millier de verrières créées ou restaurées par lui sur l'ensemble du territoire national de 1868 à son décès en $1904^{12}$. Né en 1832 à Metz, d'une famille juive bientôt installée à Paris, Hirsch est admis à l'École des Beaux-Arts en 1852 et, comme la plupart des acteurs du vitrail de sa génération, commence une carrière de peintre. L'État acquiert plusieurs des toiles qu'il expose régulièrement aux Salons sous le Second Empire, ainsi, en 1864 un Ecce Homo, envoyé à l'Hôtel-Dieu de Sète ${ }^{13}$, l'année suivante le Couronnement de la Vierge offert par Napoléon III à l'église de Leynhac (Cantal) ${ }^{14}$, en 1867 une Remise du Rosaire à saint Dominique $^{15}$, ainsi que des portraits de l'empereur, répliques de celui de Winterhalter destinées aux hôtels de ville de Besançon et de Quimperlép ${ }^{16}$. Après avoir abordé le domaine du vitrail en faisant office de cartonnier pour les peintres verriers Oudinot, Erdmann et Kremer ou Payan, il ouvre en 1868 son propre atelier de peinture sur verre, rue Gauthey, près de l'avenue de Clichy ${ }^{17}$. Dans l'intervalle deux fois marié, converti au catholicisme, il entretient les meilleurs rapports avec le clergé, ce qui lui vaut un grand nombre de commandes. Tôt introduit auprès des architectes diocésains, il crée jusqu'en 1875 vingt-trois des verrières de la cathédrale de Quimper sous la direction de Joseph Bigot $^{18}$. Ernest Massiou l'emploie en 1880 pour vitrer la cathédrale de La Rochelle ${ }^{19}$, pour laquelle il avait peint en 1870 un tableau de saint Joseph à la demande d'Antoine Brossard. Mais surtout, dès 1872, le peintre verrier devient un collaborateur régulier des chantiers de Charles Laisné, lorsque celui-ci, restaurateur de la cathédrale Sainte-Marie d'Auch, lui confie la remise en état du célèbre ensemble de vitraux du XVI ${ }^{e}$ siècle de l'édifice sous condition d'effectuer le travail sur place ${ }^{20}$ (fig. $\mathbf{n}^{\circ} \mathbf{3}$ ). Il réalise ensuite les verrières $d u$ chœur de la cathédrale de Gap, Laisné ayant pris la direction de la reconstruction du 
monument en 1876, et est appelé par lui à intervenir en restauration sur les chantiers de la cathédrale de Sens et de Notre-Dame de Brou ${ }^{21}$. Il est donc peu surprenant de retrouver des créations d'Émile Hirsch à la cathédrale de Mende, où l'architecte a été en poste jusqu'en 1874, ou au Sacré-Cœur de Paris, où il prend la succession d'Honoré Daumet en $1886^{22}$.

Figure 3

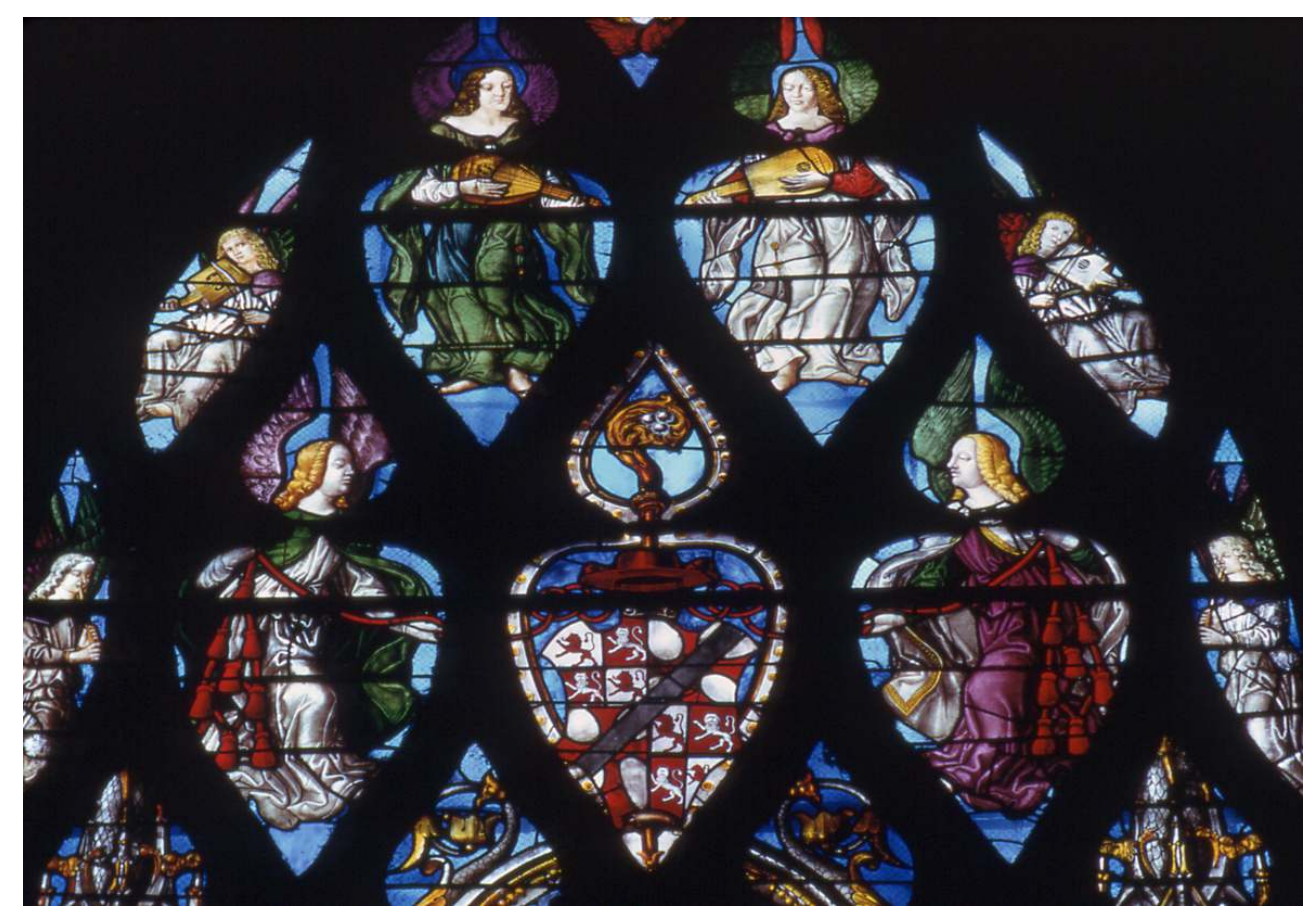

Auch, cathédrale Sainte-Marie, tympan d'une verrière réalisée vers 1510, restaurée par Émile Hirsch en 1872.

Phot. Jean Rollet. (C) Corpus vitrearum.

\section{Des images adaptées à l'institution}

6 Chacun des grands escaliers de l'École de Pharmacie s'éclaire de deux fenêtres jumelles de forme cintrée, placées en hauteur. Les ouvertures de l'escalier méridional, larges de plus de deux mètres, atteignent plus de six mètres de haut ; celles de l'escalier nord, de même largeur, sont plus courtes en raison de l'adossement extérieur de l'aile des travaux pratiques. Ainsi, hormis les bordures montées en panneaux indépendants, les armatures qui structurent les verrières, à un fer axial, délimitent quatre registres de panneaux rectangulaires sous ceux de l'amortissement du côté sud, et trois seulement en face (fig. $\mathbf{n}$ $\left.{ }^{\circ} 4\right)$. 
Figure 4

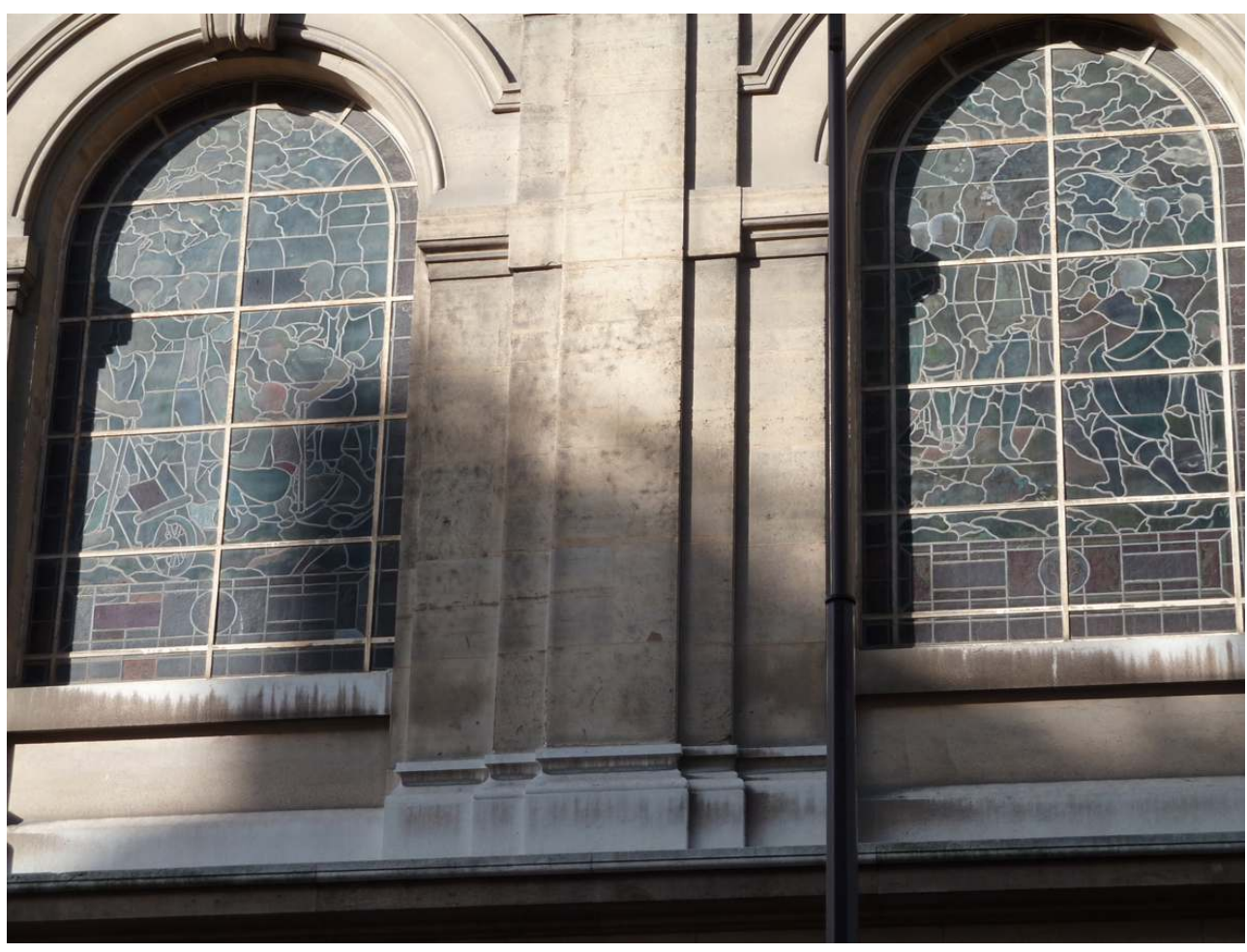

Vue extérieure des verrières de l'escalier méridional (rue Michelet).

Phot. Françoise Gatouillat.

7 Du point de vue iconographique, le projet ne mentionne que «trois scènes à sujets historiques concernant la fondation de l'institution au XVIII e siècle»; le choix de la période correspond à la vogue que connaît alors le Siècle des Lumières, qu'illustre la même année la création de la Manon de Massenet. Pour rendre hommage à des personnalités dont les travaux fondateurs ont marqué l'enseignement dispensé en ces lieux, les trois représentations, sans réelle historicité, les mettent en scène sur le mode de l'anecdote ; elles sont du reste légendées pour être comprises. D'après le cartouche de son soubassement, la verrière de gauche de l'escalier sud figure Linné reçu au Jardin des Plantes par Bernard de Jussieu (fig. $\mathbf{n}^{\circ}$ 5). Le botaniste suédois Carl von Linné (1707-1778) n’a séjourné à Paris qu'au retour d'un voyage européen, vers 1737. C'est donc à cette occasion qu'il a pu rencontrer son confrère d'origine lyonnaise Bernard de Jussieu (1699-1777), en poste à partir de 1722 au Jardin du Roi inauguré en 1640 dans le Faubourg Saint-Victor - l'actuel Jardin des Plantes -, avant que, en 1758, Louis XV ne lui confie la création de l'école de botanique des jardins de Trianon à Versailles. Le nouveau système de classification des plantes développé par ce savant, différent de celle de Linné, justifie la partie droite de l'inscription, supposée relater le dialogue entre les deux hommes, Moi aussi, je cherche à établir des familles naturelles (Linné). 
Figure 5

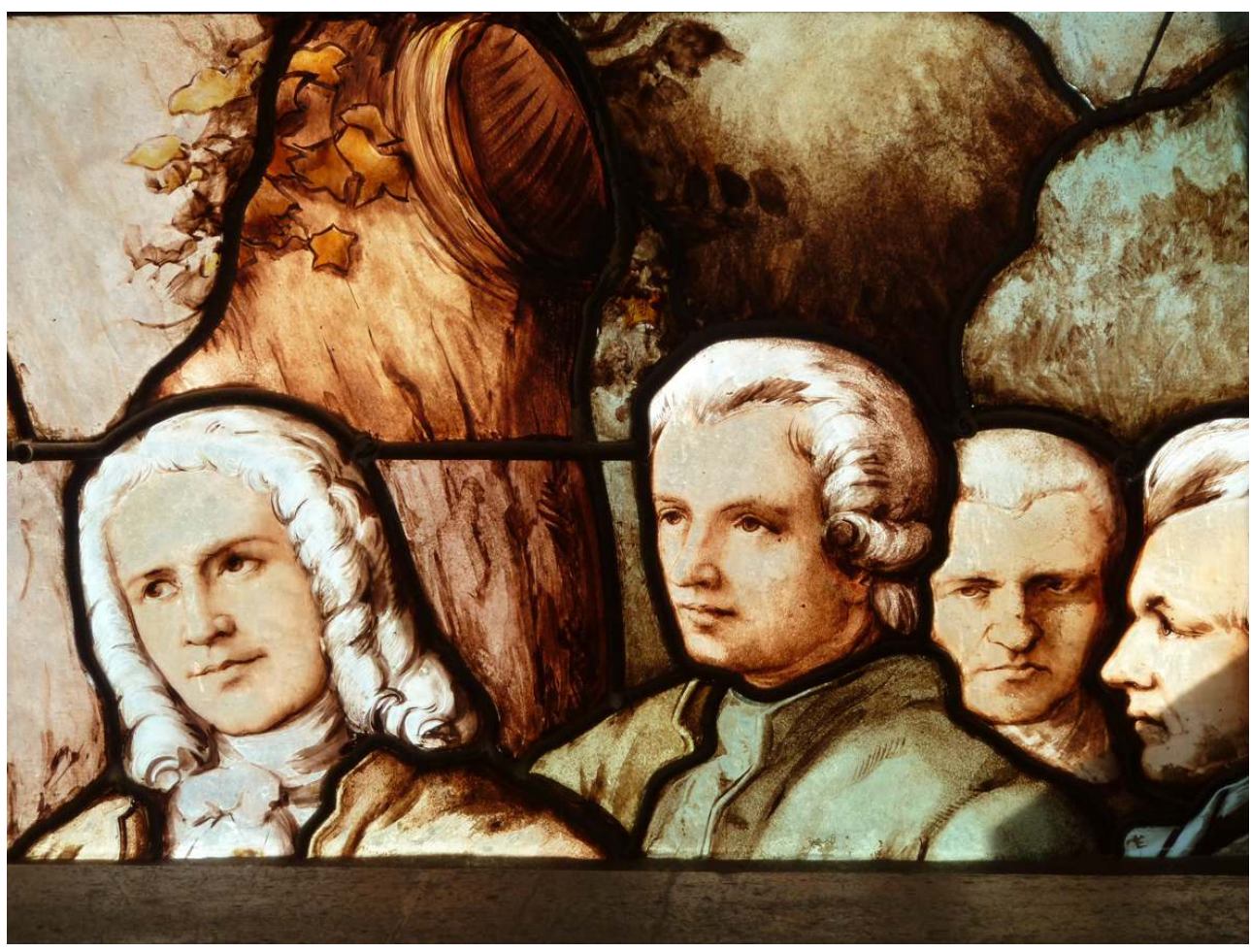

Linné reçu au Jardin des Plantes par Bernard de Jussieu, détail ; par Émile Hirsch, 1884.

Phot. Françoise Gatouillat.

8 L'autre verrière du même escalier représente le neveu de Bernard de Jussieu en une scène intitulée Laurent de Jussieu fait replanter l'École botanique; la partie droite du cartouche, Méthode naturelle Genera plantarum 1789, renvoie à l'ouvrage par lequel il a complété le système de classification des végétaux de son oncle ${ }^{23}$. Antoine-Laurent de Jussieu (1748-1836), médecin à Paris depuis 1766, nommé au poste de démonstrateur au Jardin du Roi en 1770, y enseignait la botanique avant d'être promu directeur du nouveau Muséum national d'Histoire naturelle en 1794 et d'exercer comme professeur à la Faculté de médecine de Paris jusqu'en 1826. La scène, qui se déroule sous Louis XVI devant les serres du jardin, joue sur le pittoresque horticole, peuplée d'outils, d'un arrosoir et d'une brouette ${ }^{24}$. (fig. $\left.\mathbf{n}^{\circ} \mathbf{6}\right)$ 


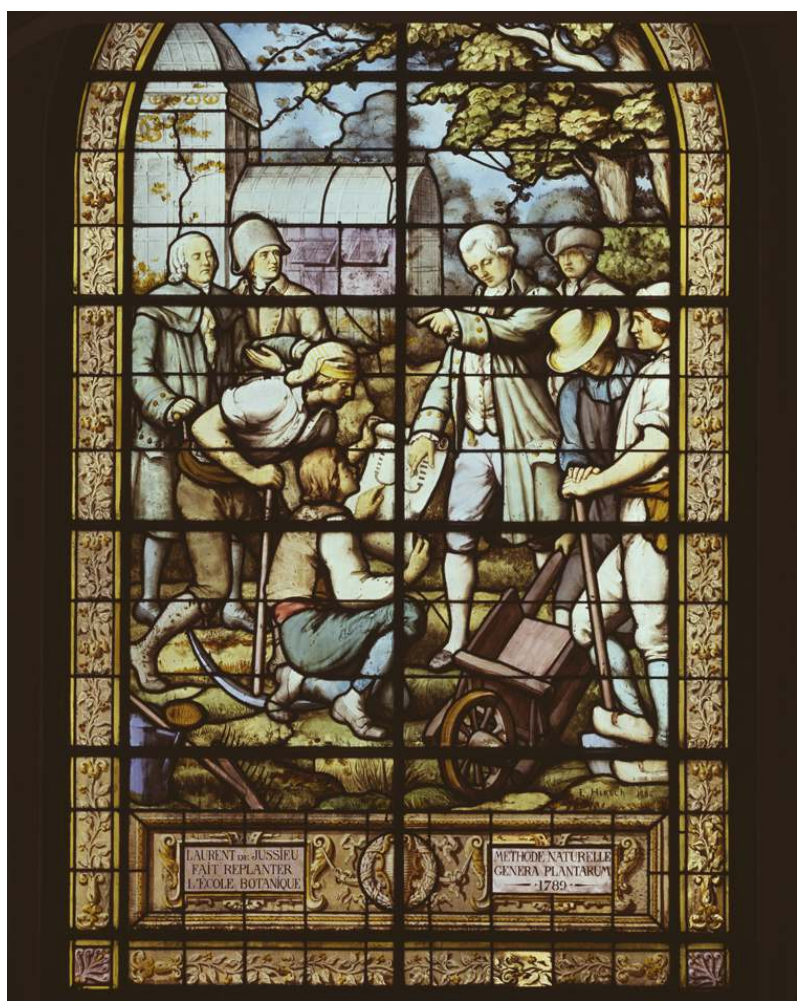

Laurent de Jussieu fait replanter l'École botanique ; par Émile Hirsch, 1884.

Phot. Philippe Fortin. (C) Inventaire général, ministère de la Culture.

Plus théâtrale encore est la verrière droite de l'escalier nord, dont la scène a pour cadre l'antre du chimiste, prétexte à l'illustration de cornues, mortiers et autres accessoires scientifiques, dans une salle où fume une grande cheminée. Le cartouche en identifie les principaux protagonistes : Lavoisier dans son laboratoire, visité par Antoine Fourcroy, Claude Berthollet et Guyton de Morveau (fig. $\left.\mathbf{n}^{\circ} \mathbf{7}\right)$ (fig. $\left.\mathbf{n}^{\circ} \mathbf{8}\right)$. Ayant isolé l'oxygène et énoncé la première version de la loi de conservation de la nature, Antoine-Laurent de Lavoisier, né en 1743, chimiste en même temps qu'économiste et philosophe, périt en 1794 sur la guillotine à cause de son office de fermier général. Ses hôtes sont ici trois confrères à peine moins célèbres. Louis-Bernard Guyton de Morveau (1737-1816), qui contribua à l'Encyclopédie de Diderot et à celle de Panckoucke pour la chimie, devint homme politique et exerça après Monge la fonction de directeur de l'École polytechnique de 1800 à 1804. Claude-Louis Berthollet (1748-1822), médecin du duc d'Orléans, entra à l'Académie des Sciences en 1780, et Antoine-François de Fourcroy (1755-1809), médecin en 1780, l'un des premiers disciples de Lavoisier, devint député à la Convention. 
Figure 7

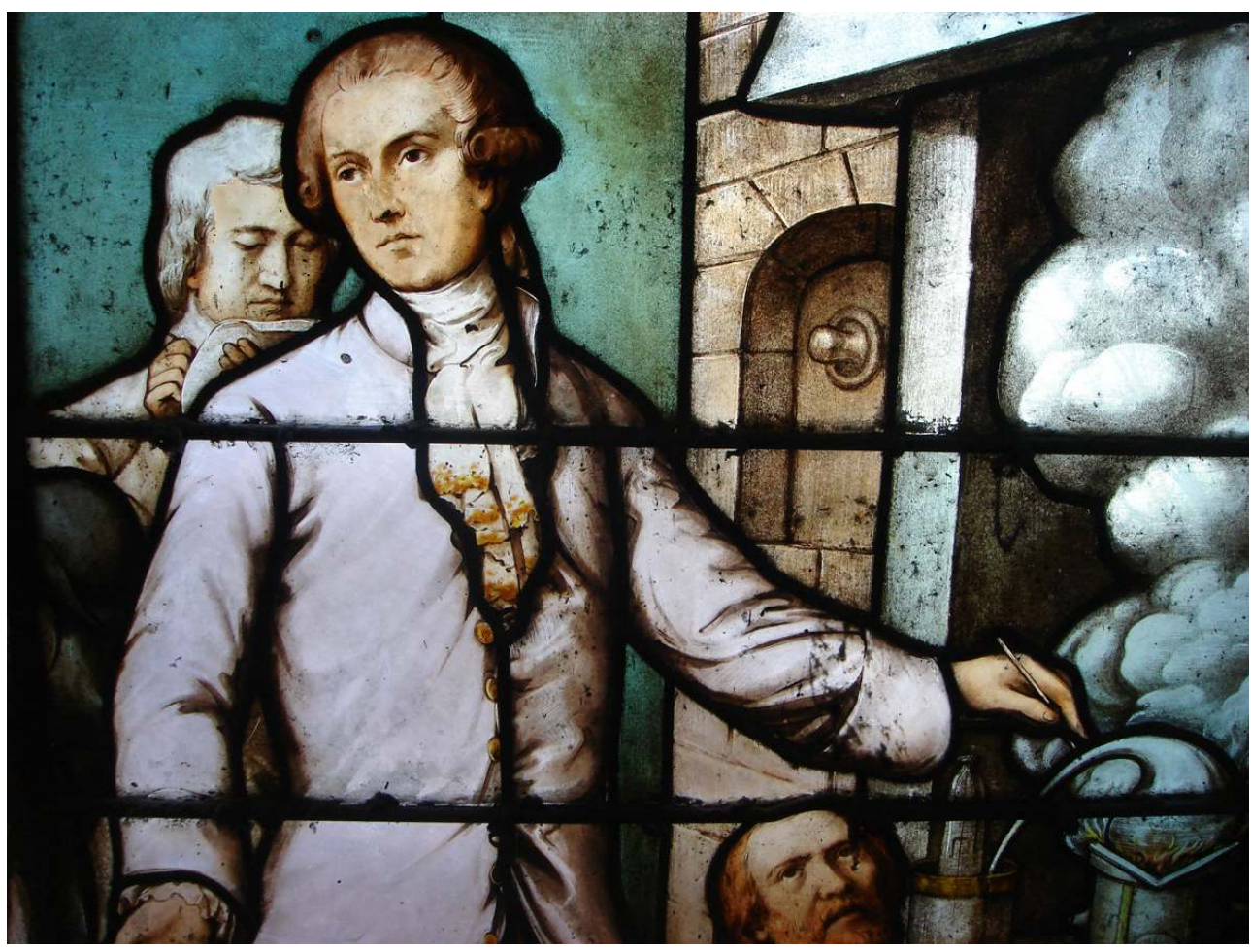

Lavoisier dans son laboratoire, détail ; par Émile Hirsch, 1884.

Phot. Françoise Gatouillat. 
Figure 8

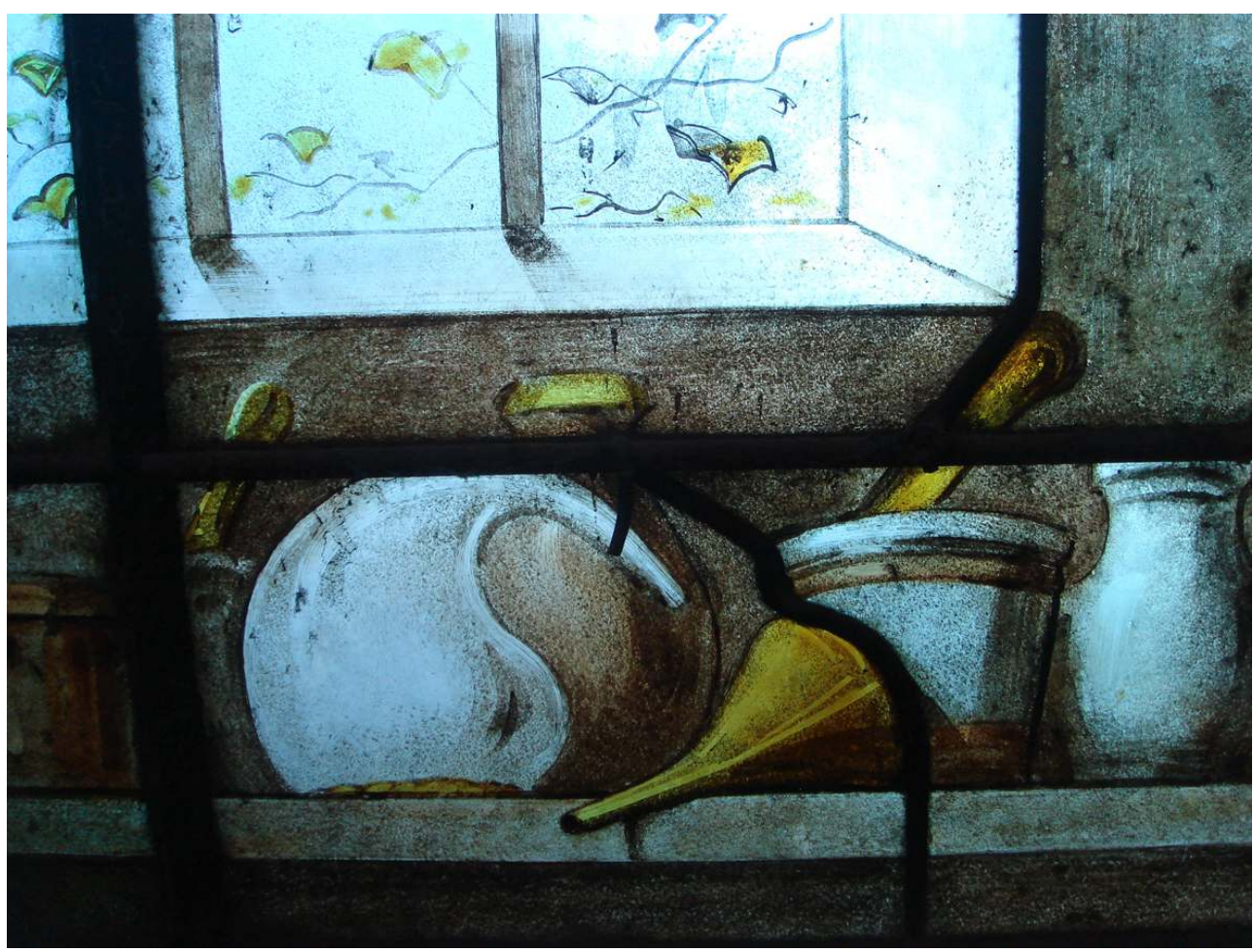

Verrière de Lavoisier, détail : les instruments du chimiste.

Phot. Françoise Gatouillat.

10 À ce programme s'ajoute, à gauche dans l'escalier nord, une représentation puisée à l'histoire du métier dans les siècles antérieurs, une réunion de l'ancienne communauté des apothicaires de Paris, associée aux épiciers depuis sa création en 1484 jusqu'aux réformes de Turgot. La scène, intitulée Corporation des Apothicaires et des Droguistes, paraît se dérouler autour de 1600 d'après le costume du porte-bannière qui occupe l'avant-plan (fig. $\left.\mathbf{n}^{\circ} \mathbf{9}\right)$. Un des membres de l'assemblée tient un parchemin sur lequel se déchiffre « $D e$ par le Roy, nous déclarons... ", texte qui paraît renvoyer à l'arrêt du Parlement du $1^{\mathrm{er}}$ août 1556 autorisant les médecins, chirurgiens et apothicaires parisiens à s'assembler « aux quatre termes de l'an et aviser entre eux à ce qui sera bon et salutaire pour le peuple ${ }^{25}$ ». Sur la bannière est peint le «blason du corps et communauté des marchands espiciers et apotiquaires espiciers de Paris » tel qu'il figure dans l'armorial général de France dressé à partir de 1696 par Charles d'Hozier : d'azur à un dextre d'argent mouvant d'une nuée de même tenant des balances d'or, coupé d'or à deux navires de gueules équipés d'azur semé de fleurs de lis d'or, flottant sur une mer de sinople et accompagné de deux étoiles de gueules. La devise de la corporation, LANCES ET PONDERA SERVANT, se lit sur le phylactère développé autour de l'écu. 
Figure 9

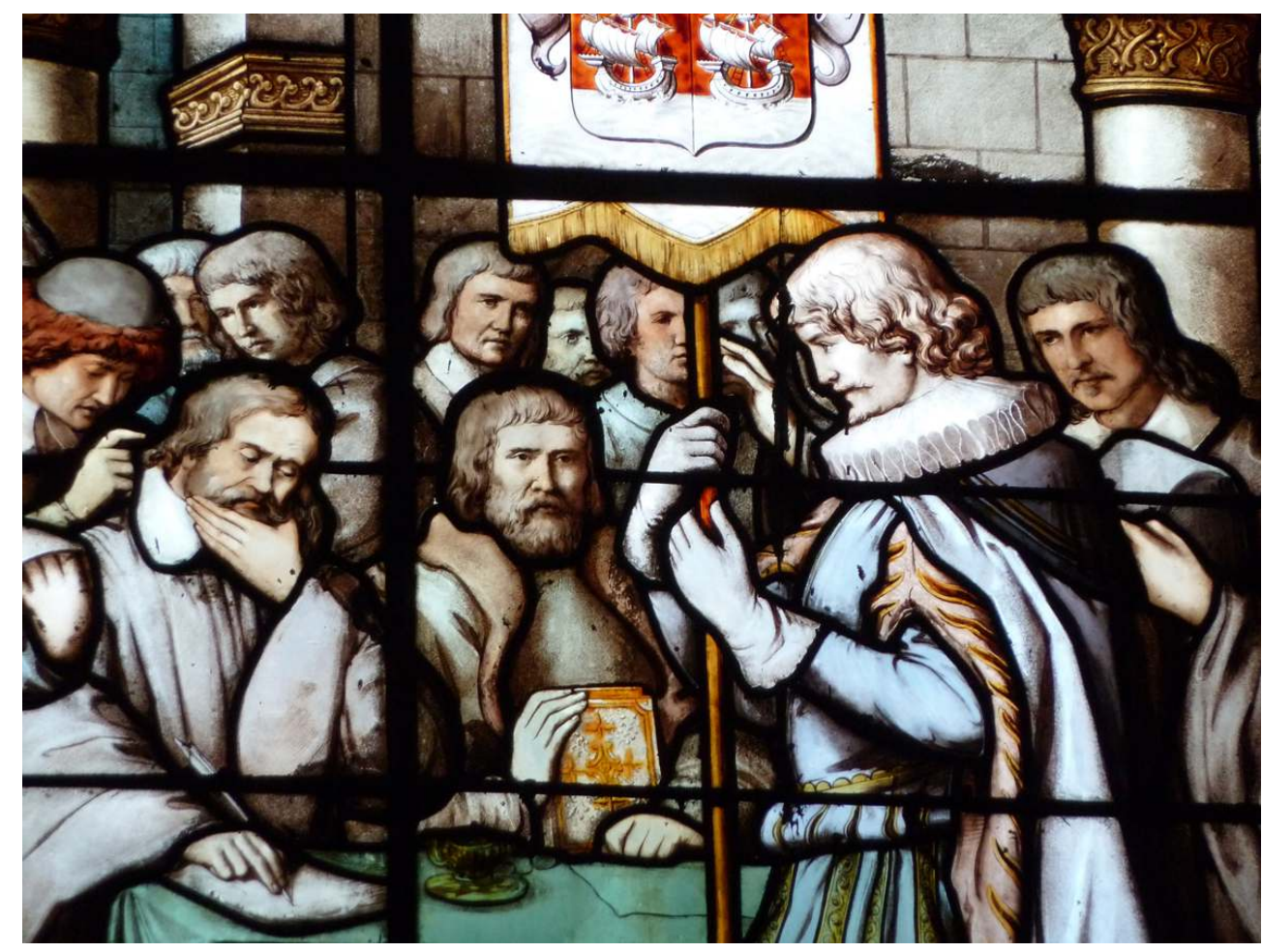

La Corporation des Apothicaires et des Droguistes, détail ; par Émile Hirsch, 1884.

Phot. Françoise Gatouillat.

\section{L'exécution}

11 Toutes quatre signées et datées de 1884, les verrières d'Émile Hirsch ne répondent pas à proprement parler au terme de « grisailles » qu'emploie régulièrement l'architecte Laisné à leur propos. Elles sont certes tirées d'une large proportion de verre blanc, mais associé à une palette de verres clairs teintés dans la masse, dominée par des nuances de rose, bleu pâle, vert et mauve. La coloration, plus vive du côté sud, compense la lumière plus franche que reçoit la façade bordée par la rue Michelet. En ce qui concerne les matériaux de peinture, l'emploi généreux de jaune d'argent et de grisailles rousses - pour les figures comme pour les bordures végétales traitées en camaïeu - donne à ces panneaux une tonalité chaude ; l'émail en est en revanche pratiquement absent (fig. $\left.\mathbf{n}^{\circ} \mathbf{1 0}\right)$ (fig. $\left.\mathbf{n}^{\circ} \mathbf{1 1}\right)$. Derrière ces réalisations de qualité se devinent des documents graphiques très élaborés. Les compositions, d'un dessin académique soigné, ont sans doute été préparées par une recherche historique, vu l'attention portée à la conformité chronologique des vêtements et des coiffures des personnages. Mais, s'il est certain que l'armorial d'Hozier a été transcrit avec exactitude sur le quatrième vitrail, il paraît moins évident que les portraits gravés des savants représentés dans les trois autres aient pu servir de modèle. Les cartouches imitant des motifs de la Renaissance ont été introduits après coup afin de réduire la surface dévolue au ciel, jugée trop développée dans la partie supérieure des premières esquisses présentées à la Commission ${ }^{26}$. 
Figure 10

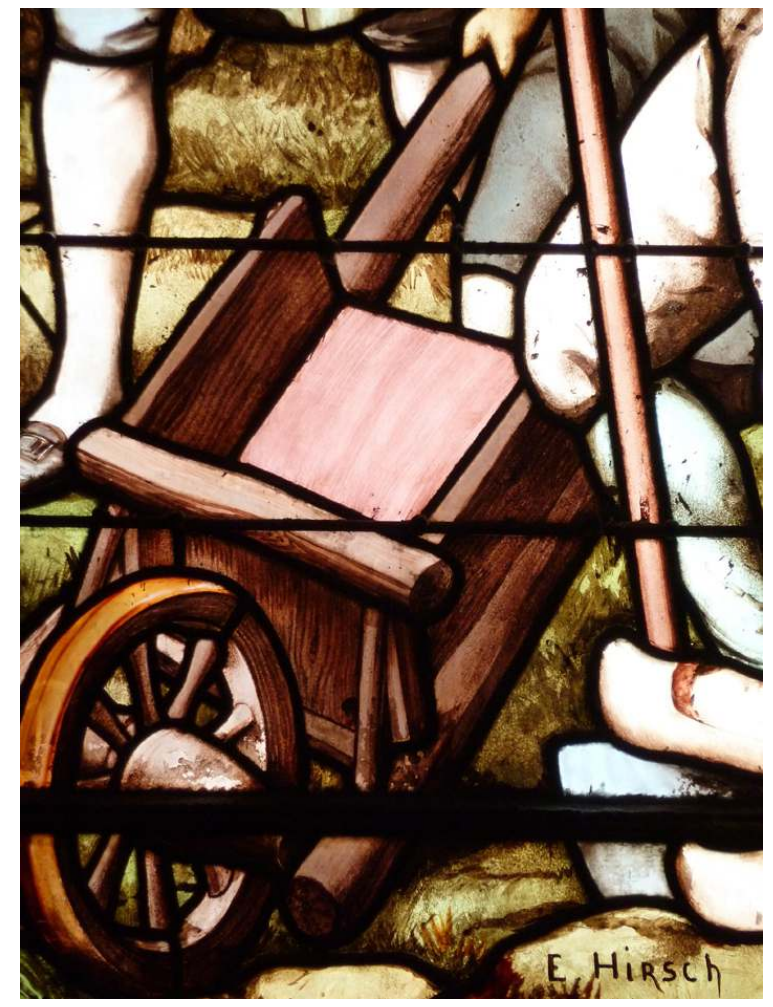

Verrières de l'escalier méridional, par Émile Hirsch, 1884 : détails de peinture. Phot. Françoise Gatouillat. 
Figure 11

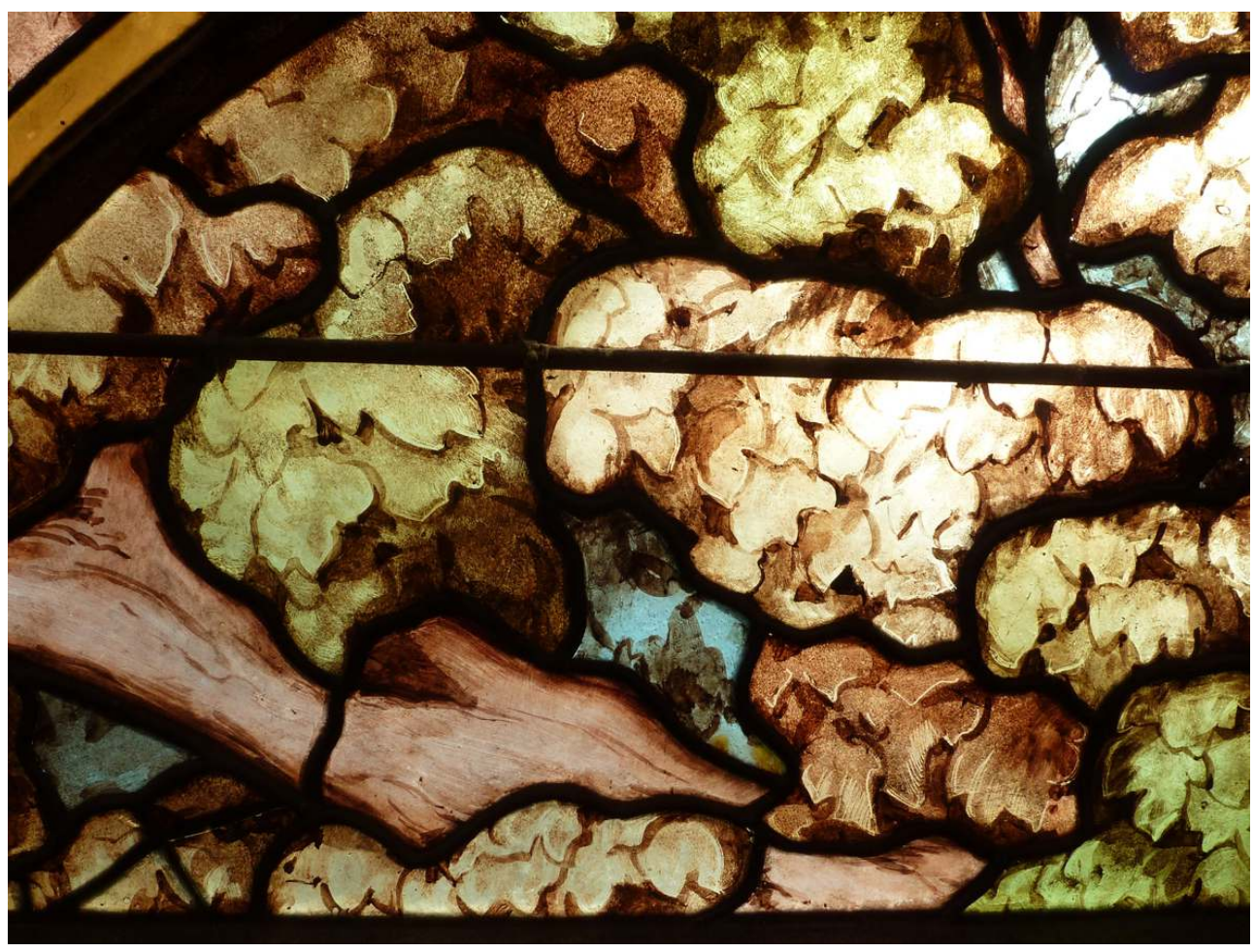

Verrières de l'escalier méridional, par Émile Hirsch, 1884 : détails de peinture.

Phot. Françoise Gatouillat.

\section{Destinées}

Depuis la modification de leur environnement architectural, l'effet de ces vitraux se trouve altéré : l'architecte Roux, chargé de surélever les bâtiments d'un étage à partir de 1932, a prolongé vers 1936 chaque escalier d'une nouvelle volée de marches, qui passent devant les verrières et les obstruent en partie : au nord comme au sud, l'une des verrières est recoupée par le palier intermédiaire (fig. $\mathbf{n}^{\circ} \mathbf{1 2}$ ), et l'amortissement de sa voisine se trouve dissimulé. Des travaux d'une telle ampleur n'ont pu être effectués sans que les vitraux soient mis à l'abri, et les restaurations décelables en certains endroits - par exemple la moitié droite du cartouche du vitrail des apothicaires - ont probablement été pratiquées pendant cette campagne. Les cartons utilisés pour les quatre réalisations ont été attribués au musée des Arts et Industries de Saint-Étienne (Loire) par décision ministérielle du 17 janvier $1890^{27}$. Ils n'y sont pour l'instant plus localisés mais le dernier récolement des collections des deux musées de la ville, en août 2008, a néanmoins fait apparaître des photographies des verrières en place, d'un intérêt certain parce que prises avant le remaniement des cages d'escaliers ${ }^{28}$. 
Figure 12

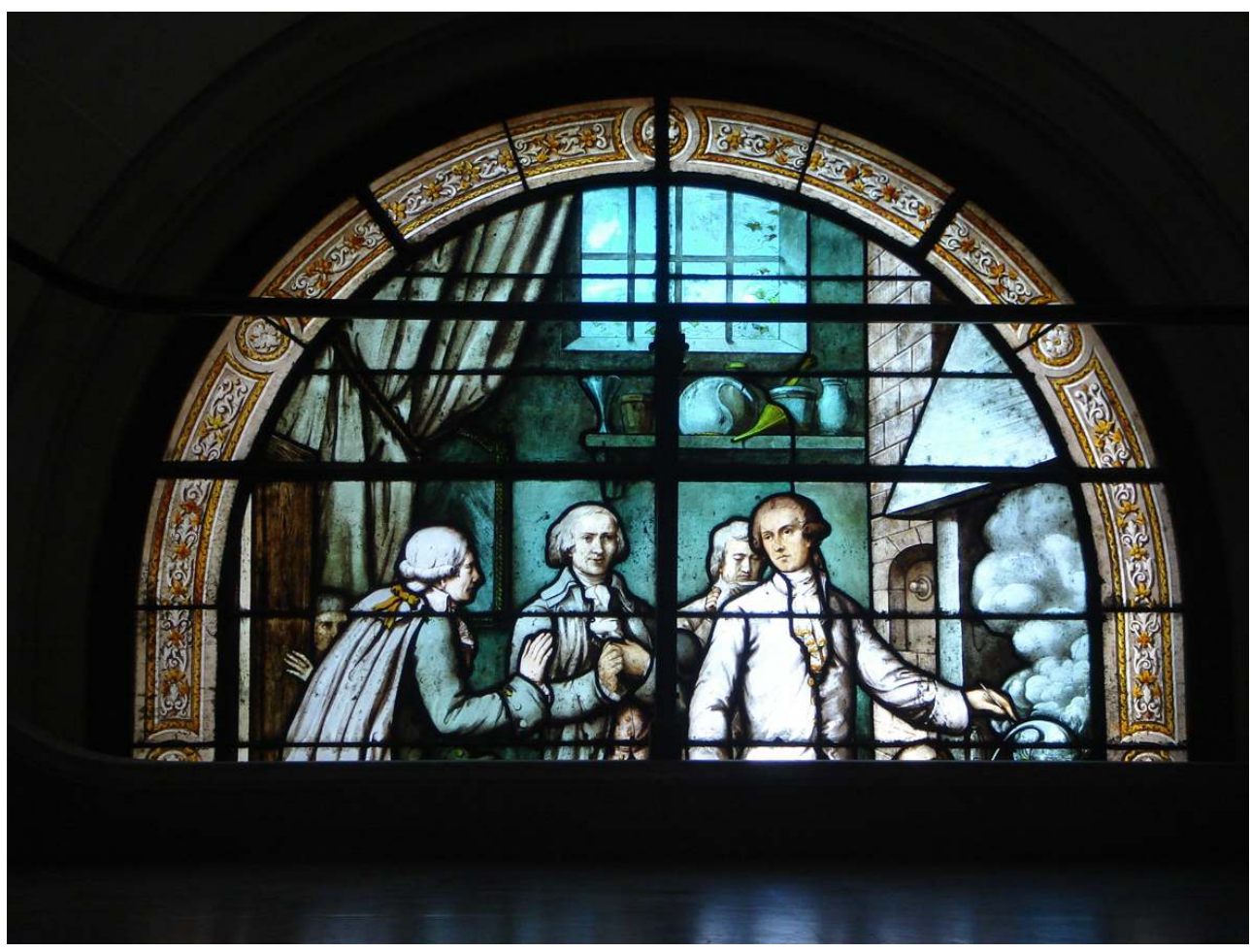

Lavoisier visité par Antoine Fourcroy, Claude Berthollet et Guyton de Morveau ; par Émile Hirsch, 1884. Scène coupée depuis la réfection de l'escalier vers 1936.

Phot. Françoise Gatouillat.

\section{Un décor complété}

Le décor du vestibule d'honneur fait bientôt l'objet d'une nouvelle campagne, qui compte notamment quatre grandes toiles d'Albert Besnard datées de 1888, L'excursion botanique, L'excursion géologique, Le cours de chimie et Le cours de physiologie ${ }^{29}$. Le projet que Charles Laisné avait défini en 1883 pour la grande verrière du fond de la salle est repris en 1887, précisément l'année du décès de Claudius Lavergne, le peintre verrier initialement pressenti. Comte, nouveau directeur des Bâtiments civils, est alors saisi de la demande de l'architecte: «Le grand vestibule... de l'École est décoré dans sa partie intérieure de peintures de M. Besnard ayant trait à l'art de guérir dans les temps modernes. Mais le fond de ce vestibule réclame quelque coloration pour le relier aux peintures de l'entrée. Pour obtenir cette harmonie désirable, j'aurais l'honneur de vous proposer de décorer par un vitrail la grande croisée qui s'ouvre sur le jardin. Ce vitrail pourrait rappeler les origines de la Médecine et de la Pharmacie en représentant le temple d'Esculape à Épidaure, où d'une part les prêtres recevaient les malades et y inscrivaient sur des tablettes leurs ordonnances, et d'autre part où les malades apportaient leurs offrandes à la divinité du lieu. L'exécution de cette verrière pourrait être confiée à un de nos peintres verriers les plus estimés, $M$. Hirsch, qui a déjà décoré de grisailles les baies des escaliers ${ }^{30}$ ». La suggestion de l'architecte concernant l'iconographie n'est pas retenue: le sujet adopté est finalement plus solennel, réunissant l'allégorie de la Pharmacie flanquée de celles de la Botanique et de la Chimie. Son choix du peintre verrier est en revanche validé, 
en dépit des réticences émises par le Comité des Travaux d'art qui, à l'occasion d'une inspection des nouvelles peintures de Besnard, n'a guère apprécié « les vitraux d'un des escaliers». Émile Hirsch, soutenu par Laisné et, d'après une lettre souvent citée, manifestement familier du ministre lui-même ${ }^{31}$, obtient la commande le 29 mai 1887, non sans avoir remanié suivant les indications du Comité la maquette qu'il lui avait présentée ${ }^{32}$. Laisné et l'inspecteur des Beaux-Arts Roger-Ballu approuvent ses cartons à grandeur d'exécution le 7 mars 1888, prélude à l'exécution de la verrière, achevée en juin pour le prix de $6100 \mathrm{~F}$. Le peintre verrier est ensuite invité à livrer ses cartons, propriété de la direction des Beaux-Arts qui en a assuré la dépense, au dépôt de lî̂le des Cygnes situé au 182 , rue de l'Université.

La verrière elle-même a eu moins de chance encore que celles des escaliers: la construction d'un nouveau bâtiment en arrière du vestibule vers 1960 a entraîné sa dépose, la fenêtre étant supprimée et remplacée par une porte. L'œuvre est remisée en caisse depuis un demi-siècle dans les caves de l'établissement. Une photographie de groupe prise au début du XXe siècle dans le hall permet toutefois d'apercevoir ce vitrail monumental, dans lequel on devine les trois allégories en pied abritées d'un grand frontispice de style Renaissance ${ }^{33}$; les neuf panneaux sur lesquels sont figurés les trois personnages ont d'autre part été présentés en 1999 dans l'exposition Les palais de la science tenue à la Marie du Ve arrondissement (voir note 6).

\section{Projets inaboutis}

Dès janvier 1889, au prétexte qu'il ne s'harmonise guère avec les peintures de Besnard, Roger-Ballu, qui avait pourtant soutenu l'entreprise, demande le retrait de ce vitrail tout neuf ${ }^{34}$. Le complément du décor vitré, envisagé courant 1888 , échappera en tout cas à Hirsch. Charles Laisné conçoit en effet de nouveaux projets pour le vestibule d'honneur, l'ajout de $120 \mathrm{~m}^{2}$ de peintures supplémentaires à ses parois et à sa voûte, et d'une série de vitraux à ses fenêtres latérales (fig. $\mathbf{n}^{\circ} \mathbf{1 3}$ ). Fort d'une promesse d'Edmond Turquet, soussecrétaire d'État aux Beaux-Arts, et de Jules Castagnary, président du Conseil municipal de Paris, Albert Besnard dessine fin 1888 huit cartons à sujets animaliers pour ces fenêtres, d'un esprit si différent de celui de la verrière qu'Émile Hirsch vient de poser, qu'ils justifient l'insolite requête de Roger-Ballu: le décorateur propose un séduisant hommage à la faune et à la flore universelles en des paysages naturalistes hautement colorés, fort variés mais unifiés par d'étroits encadrements architecturaux à pilastres traités en perspective. Fenêtres fictives destinées à s'intégrer au bas des vantaux des baies réelles, elles ouvrent sur des aigles au soleil couchant, des cygnes évoluant au bord d'un lac, un singe dans la jungle, des autruches environnées de cannes à sucre, une cascade, des paons, des ruches devant un champ de blé survolé par des moineaux, et une bassecour où se pressent un dindon, des oies, un coq, des poules et des pigeons (fig. $\left.\mathbf{n}^{\circ} \mathbf{1 4}\right)$. Divisés chacun en deux pans verticaux pour constituer un tableau carré d'environ 1,60 m de côté, les documents préparatoires portent tous les détails utiles à l'exécution, le passage des plombs et des barlotières ainsi que l'emplacement des vergettes. Georges Rouault, apprenti peintre verrier depuis l'âge de 14 ans, puis employé de 1887 à 1890 chez Hirsch, aurait décliné par loyauté envers ce dernier l'offre de Besnard de prendre part à la réalisation des vitraux ${ }^{35}$. Quoi qu'il en soit, le ministère des Cultes et des Beaux-Arts ayant refusé d'accorder le budget nécessaire, les ultimes propositions de Laisné sont 
abandonnées en 1890 et les fenêtres du hall demeurent définitivement closes de simples vitres.

Figure 13

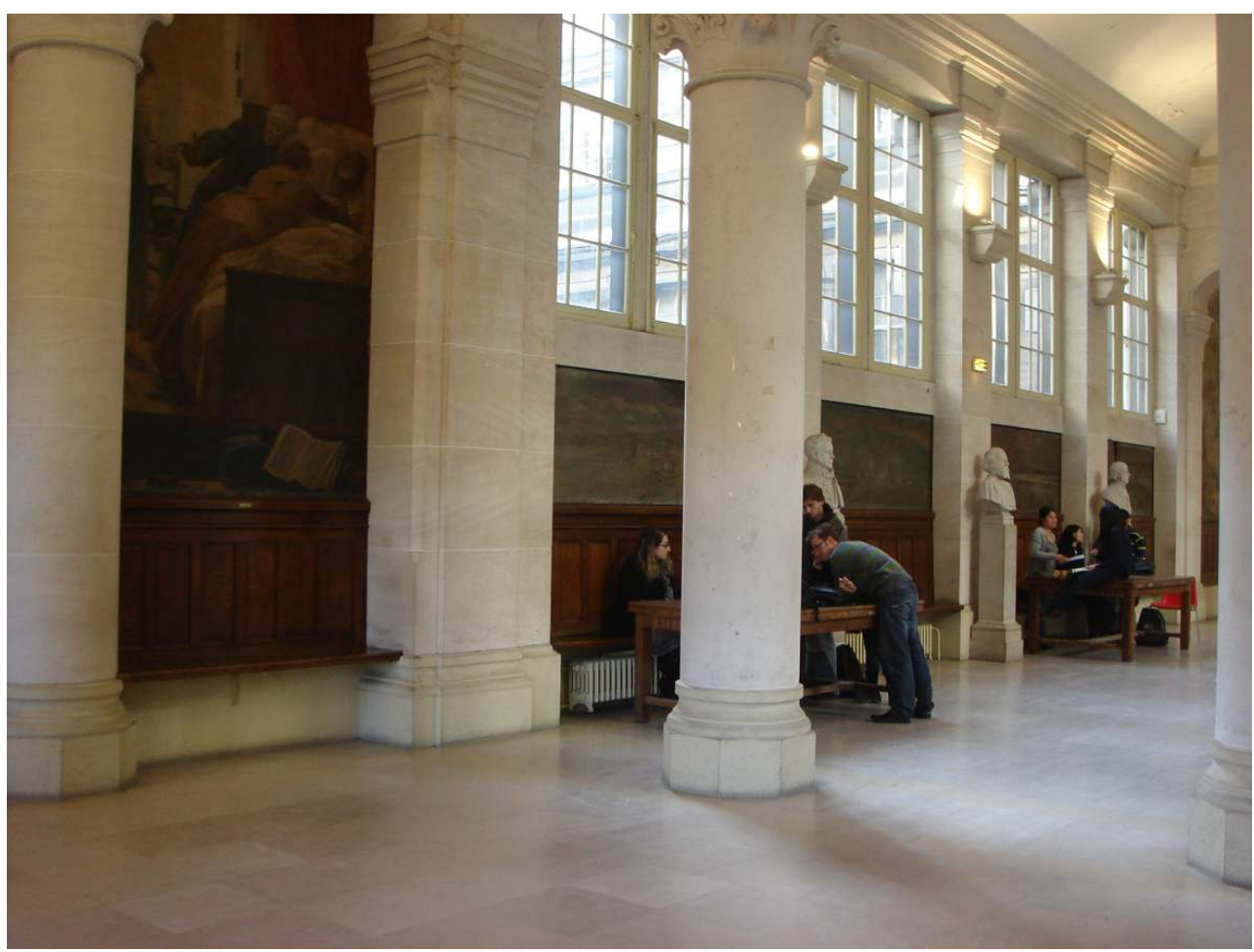

Les fenêtres latérales du vestibule d'honneur, côté sud.

Phot. Françoise Gatouillat. 


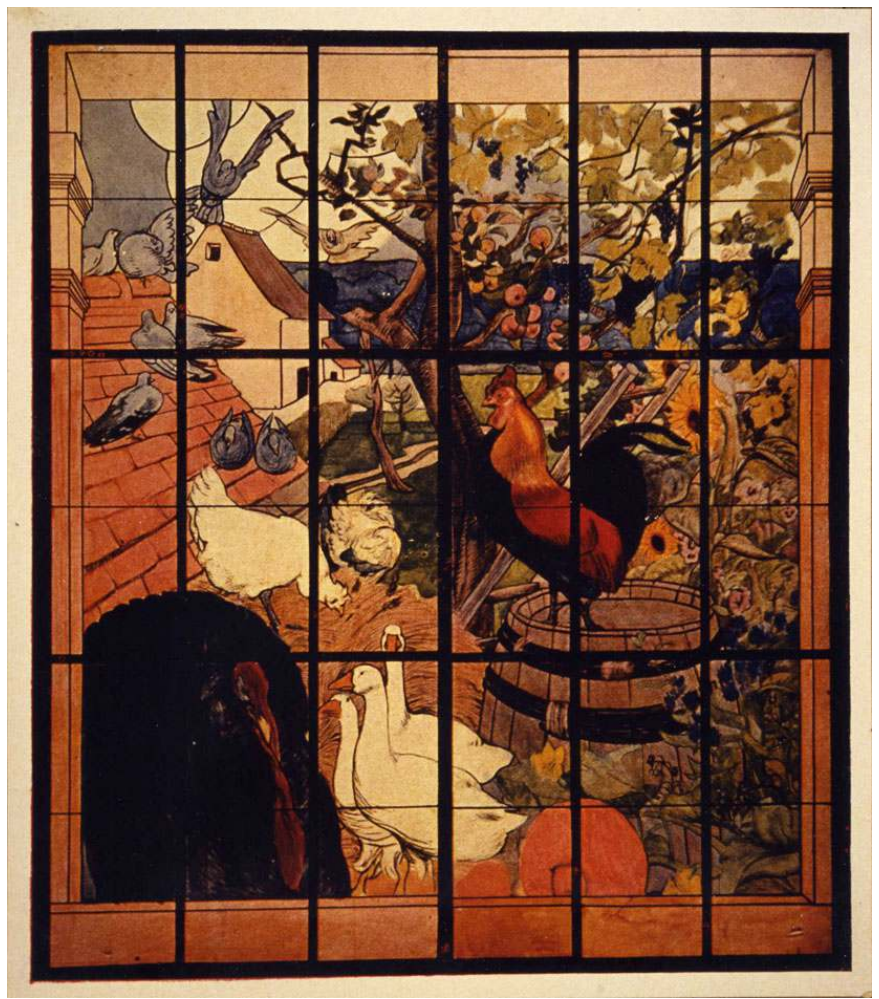

La basse-cour, carton d'Albert Besnard pour l'une des fenêtres latérales du vestibule d'honneur. Phot. tirée de la revue Art et Industrie, 1910.

L'aventure a toutefois le mérite de mettre en lumière, à ce moment précis de l'histoire de la commande publique, la coexistence de systèmes esthétiques radicalement antagonistes. En offrant une alternative aux formules de l'histoire rétrospective et de l'allégorie, devenues banales dans l'art public depuis le Second Empire, les projets de vitraux de Besnard, dépourvus de toute icône historique ou mythologique, représentent une voie d'avant-garde, d'autant qu'ils émanent « d'un artiste qui n'aurait pas manqué de jeter un coup d'œil vers le Japon ${ }^{36} »$. Ces cartons, caractéristiques de l'Art nouveau désormais au goût du jour, ont d'emblée connu une certaine notoriété, montrés dans des expositions au cours des deux décennies suivantes, commentés et reproduits dans les revues spécialisées ${ }^{37}$. À l'occasion de leur présentation au Pavillon de Marsan en 1910, il est constaté que le temps en a déjà altéré les couleurs ${ }^{38}$. L'ensemble des cartons, en effet en médiocre état, est resté conservé dans les fonds du Musée des Arts décoratifs, où l'État l'a mis en dépôt en avril $1892^{39}$.

Postérité inattendue du décor de l'École de Pharmacie, deux des cartons d'Albert Besnard ont été transcrits sur verre par Henri Carot ${ }^{40}$. L'un, les Cygnes sur le lac d'Annecy, a été réalisé en 1890 pour le vestibule de la résidence parisienne du peintre Henry Lerolle, un hôtel situé 20 , avenue Duquesne (fig. $\mathbf{n}^{\circ}{ }^{15}$ ), (fig. $\mathbf{n}^{\circ} \mathbf{1 6}$ ). L'encadrement en camaïeu de la composition a été supprimé pour augmenter la surface colorée, et la compartimentation initialement prévue a été réduite à neuf panneaux; du point de vue du traitement pictural, la grisaille vient simplement ponctuer les effets des verres plaqués gravés à l'acide qui traduisent l'eau et les plantes, et les verres à reliefs utilisés pour le plumage des animaux (fig. $\mathbf{n}^{\circ}{ }^{17}$ ). Ce vitrail de haute qualité a été offert en 1938 au musée des Arts 
décoratifs par les fils de l'artiste ; attribué au musée d'Orsay depuis 1981, il est exposé dans la section Art nouveau ${ }^{41}$. À l'initiative de l'érudit et philanthrope Jules Maciet et de Georges Berger, président de l'Union centrale des Arts décoratifs, Carot a également réalisé les Paons, en adaptant le carton à la forme d'une baie cintrée, de dimensions supérieures à celles de l'École (H. 3,12 m - L. 2,49 m). L'œuvre, elle aussi remarquable, actuellement entreposée dans les réserves du musée, a figuré au Salon dissident de $1895^{42}$. La même année, le verrier américain Louis Comfort Tiffany se serait d'autre part inspiré du carton de la Cascade de Besnard ${ }^{43}$.

Figure 15

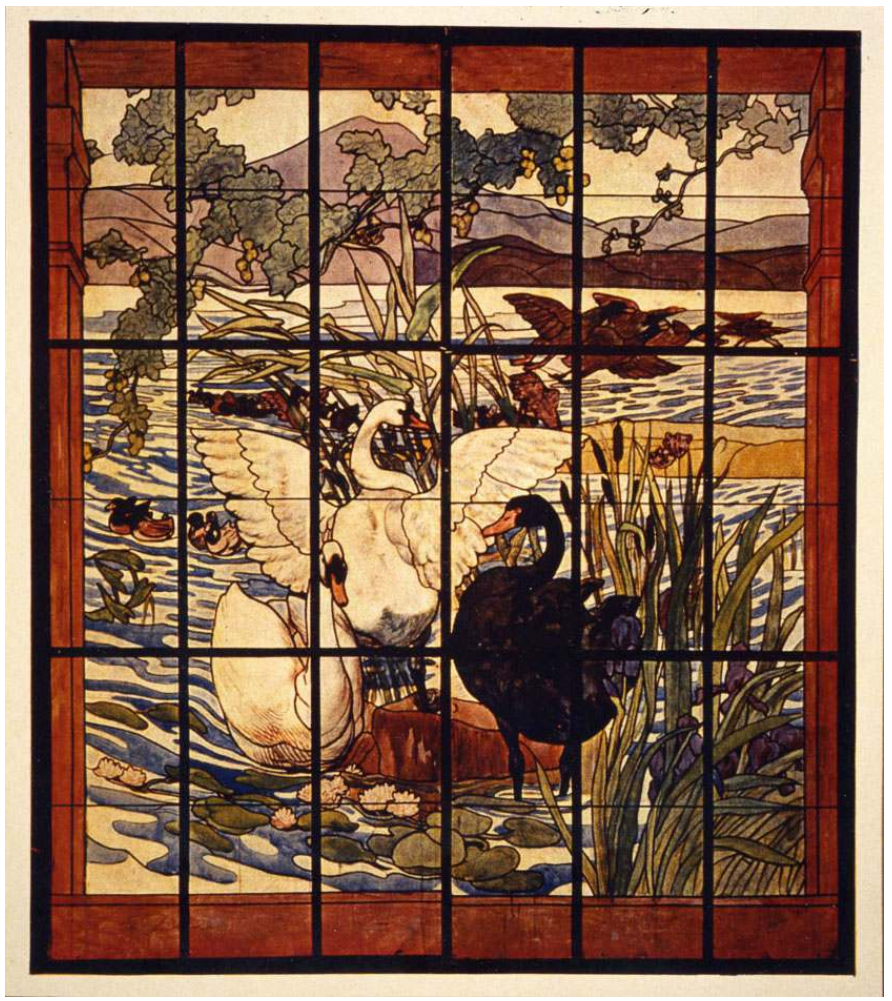

Les cygnes sur le lac d'Annecy, carton d'Albert Besnard pour l'une des fenêtres latérales du vestibule d'honneur.

Phot. tirée de la revue Art et Industrie, 1910. 
Figure 16

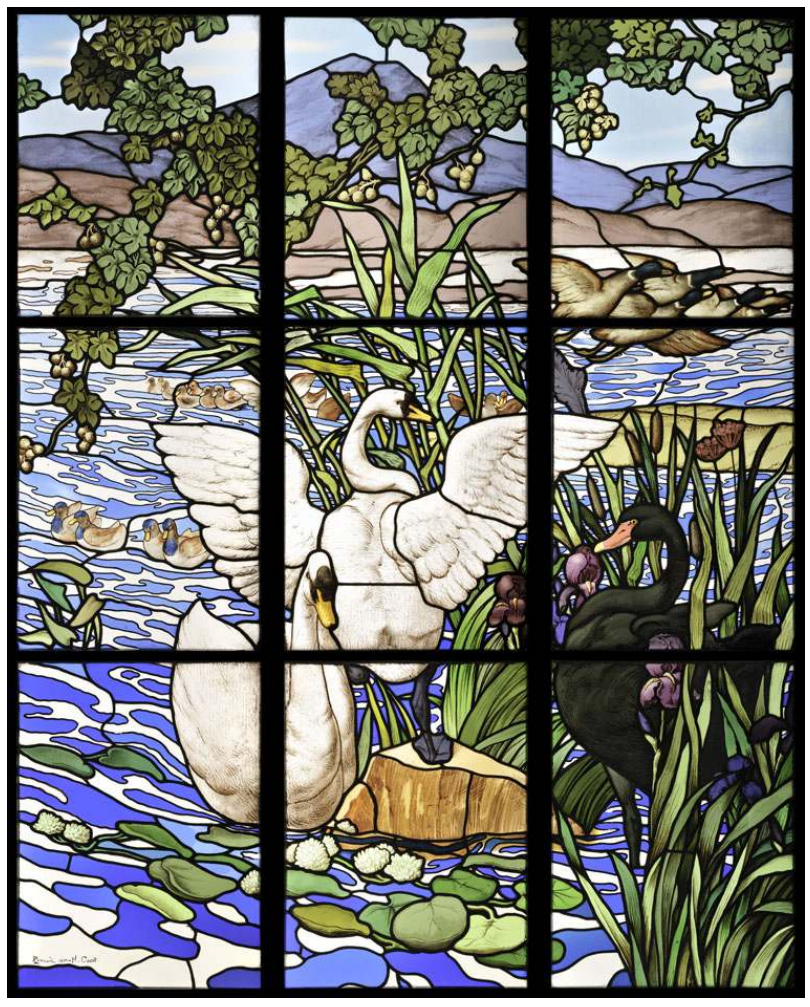

Les cygnes sur le lac d'Annecy, vitrail réalisé en 1890 par Henri Carot sur les cartons d'Albert Besnard. Phot. Stéphane Maréchalle/René-Gabriel Ojéda. (c) RMN (Musée d'Orsay). 


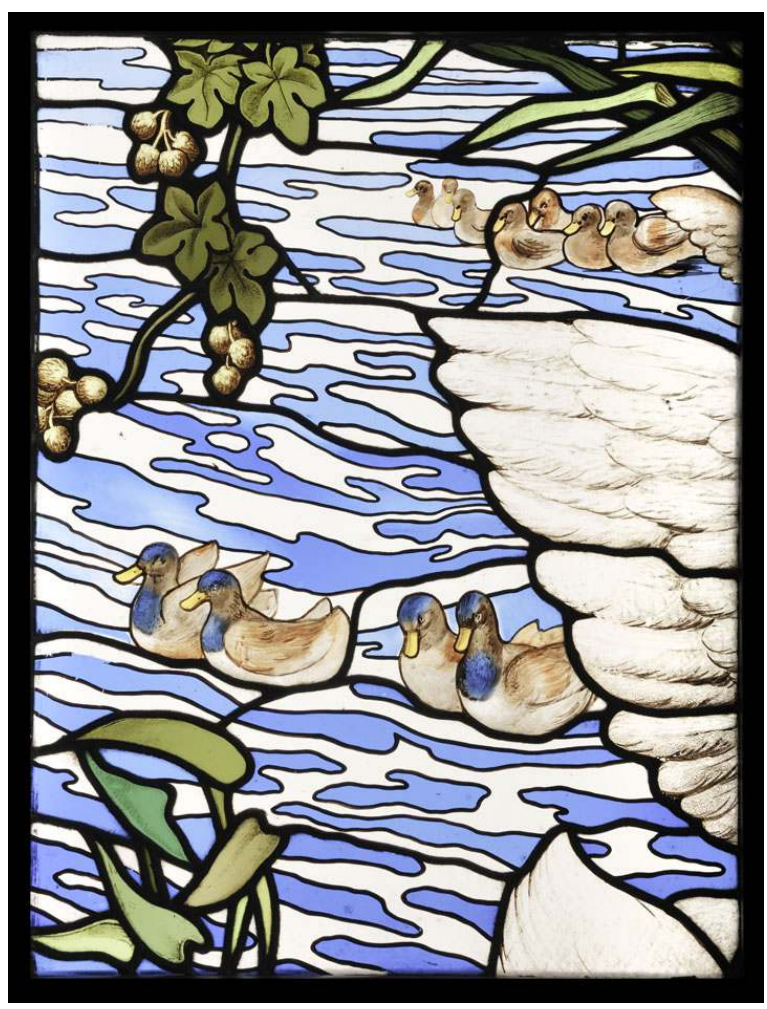

Les cygnes sur le lac d'Annecy, détail : verres gravés.

Phot. Stéphane Maréchalle/René-Gabriel Ojéda. (C) RMN (Musée d'Orsay).

\section{NOTES}

1. - Voir FINANCE, Laurence de. «Le vitrail civil». Dans Un patrimoine de lumière, 1830-2000. Verrières des Hauts-de-Seine, Seine-Saint-Denis, Val-de-Marne. Paris : Monum, Éditions du patrimoine, 2003, p. 153-169 (coll. Cahiers du Patrimoine, nº 67).

2. - Sorbonne, Palais de Justice, Panthéon, mairies d'arrondissements, etc.

3. - LUNEAU, Jean-François. Félix Gaudin peintre-verrier et mosaïste (1851-1930). Clermont-Ferrand : Presses universitaires Blaise Pascal, 2006, p. 299.

4. - VALETTE, Simonne. « Les richesses artistiques de la Faculté de Pharmacie ». Revue d'Histoire de la Pharmacie, $\mathrm{n}^{\circ} 177,1963$, p.104. VALETTE, Simonne. «Ce qu'il faut voir à la Faculté de Pharmacie de Paris ». Revue de la Société d'Histoire de la Pharmacie, 1973, n²18, p. 539. VALETTE, Simonne. «Les richesses artistiques de la Faculté de Pharmacie ». Dans La Faculté de Pharmacie, 1882-1982. Saint-Cloud, 1982, p. 62-77. BZOURA, Élie. « Le patrimoine de la faculté de Pharmacie de l'Université Paris V ». Revue d'Histoire de la Pharmacie, vol. 86, $2^{\mathrm{e}}$ trimestre 1998, p. 238-250. LE BRIZE, Sandrine. Richesses et patrimoine de l'université Paris Descartes, janvier 2009, p. 21-32 (www. univ-Paris5.fr/fre/).

5. - Base ELEC de l'École des chartes. 
6. - HOTTIN, Christian. Quand la Sorbonne était peinte. Étude sur la décoration peinte et sculptée des établissements d'enseignement supérieur parisiens. Thèse de l'École des chartes, 1997, vol. VI, p. 522-572 (Arch. nat., AB XXVIII 1126 et 1127 (12 vol.)). DOULAT, Fabienne. «L'École supérieure de pharmacie ». Dans HOTTIN, Christian (dir.). Universités et grandes écoles à Paris. Les palais de la science. Paris, 1999, p. 116-120.

7. - Arch. nat., $\mathrm{F}^{21} 4867$.

8. - HEUVRARD-BEAUVALOT, Chantal. Albert Besnard (1849-1934) : une vocation de décorateur. Thèse, université de Paris X Nanterre, dir. Ségolène Le Men, 2005, p. 123-124. L'artiste présente au Salon de 1884 deux de ses toiles, la Maladie et la Convalescence, avant leur marouflage autour de la porte de la salle des Actes (Base ARCHIM, photographies des œuvres acquises par l'État exposées aux Salons, $\left.\mathrm{F} / 21{ }^{*} 7654,1884, \mathrm{n}^{\circ} 212\right)$. D'autres sujets, la Cueillette des simples, le Traitement des simples et le Laboratoire portent la date de 1885.

9. - Arch. nat., $\mathrm{F}^{21}$ 4867, lettre de Laisné au ministre, 16 avril 1883.

10. - Sur ces verrières de 1878, déposées ou détruites en 1936, voir L'exposition universelle de Paris, 1878. Catalogue officiel. Paris, 1878, vol. II, p. 71-73, 101-102 et 109-112. Hirsch était l'auteur d'une allégorie de la peinture et d'une verrière de la série des Arts industriels, L'orfévrerie; pour cette dernière, il avait été préféré à Joseph Félon, dont la maquette est conservée (Collection particulière, le goût d'un amateur bordelais, catalogue d'exposition, Bordeaux, 2008).

11. - Arch. nat., $\mathrm{F}^{21} 4867$, courriers du 9 mai au 21 juin 1883.

12. - HARTMANN, Paul. Émile Hirsch peintre-verrier, 1832-1904. Brochures multigraphiées, Sceaux, 1987, 1990.

13. - Base ARCHIM, album de photographies des Salons, $F / 21 /^{*} 7635$ ( $\left.n^{\circ} 953\right)$.

14. - Base Palissy.

15. - Base ARCHIM, album de photographies des Salons, F/21/*7638 ( $\left.\mathrm{n}^{\circ} 755\right)$.

16. - Arch. nat., $\mathrm{F}^{21} 147$.

17. - HARTMANN, Paul. Émile Hirsch peintre-verrier, 1832-1904. Brochures multigraphiées, Sceaux, 1987, 1990.

18. - BONNET, Philippe. Quimper, la cathédrale. Paris : Zodiaque, 2003, p. 67 et passim.

19. - Base Palissy, entre autres Saint Louis (baie 113), cl. A. Maulny, 88171825XA. Voir MAILLES, Dominique et RIOU, Yves-Jean. La Cathédrale Saint-Louis de La Rochelle. Poitiers : Connaissance et promotion du patrimoine de Poitou-Charentes, 1985 (coll. Images du Patrimoine, $n^{\circ} 12$ ).

20. - COUTURE, Léonce. « Restauration des vitraux de la cathédrale d'Auch ». Revue de Gascogne, $\mathrm{t}$. XIV, 1873, p. 382-385.

21. - À Sens, Hirsch restaure les vitraux de la face orientale des bras du transept et plusieurs chapelles du chœur entre 1881 et 1884 . Les vitraux de Bourgogne, de Franche-Comté et de Rhône-Alpes. Corpus vitrearum, Recensement III, 1986, p. 175 ; les travaux effectués à Brou sont à peu près contemporains (ibid., p. 250).

22. - Archives privées : Hirsch, listes manuscrites de ses travaux personnels, 1896.

23. - JUSSIEU, Antonii-Laurentii de. Genera plantarum secundum ordines juxta methodum in Horto regio Parisiensi exaratam anno MDCCLXXIV. Paris : Veuve Hérissant, 1789.

24. - Un détail de la verrière est reproduit dans l'ouvrage de BLONDEL, Nicole. Le vitrail: Vocabulaire typologique et technique. Paris: Imprimerie nationale, 1993, n 234, p. 133 (coll. Principes d'analyse scientifique).

25. - Je remercie amicalement Benoît Dufournier pour la documentation qu'il m'a fournie.

26. - Arch. nat., $\mathrm{F}^{21} 2087$ (dossier Hirsch), février 1884.

27. - Arch. nat., $\mathrm{F}^{21} 2087$.

28. - Mes remerciements s'adressent à la conservation des deux musées de Saint-Étienne et à Xavier-Philippe Guiochon pour les recherches qu'ils ont bien voulu faire à ma demande.

29. - HEUVRARD-BEAUVALOT, Chantal. Albert Besnard (1849-1934): une vocation de décorateur. Thèse, université de Paris X Nanterre, dir. Ségolène Le Men, 2005, p. 124. 
30. - Arch. nat., $\mathrm{F}^{21} 4867$, courriers des 14,21 et 28 mars 1887, joints à une copie du devis de Laisné.

31. - « M. le Ministre, m'as-tu oublié ? J’attends toujours la commande des vitraux du vestibule de l'École de Pharmacie» (Voir VALETTE, Simonne. "Les richesses artistiques de la Faculté de Pharmacie ». Dans La Faculté de Pharmacie, 1882-1982. Saint-Cloud, 1982, p. 72).

32. - Arch. nat., $\mathrm{F}^{21} 4867,25$ mai et 11 juin 1887 ; $\mathrm{F}^{21}$ 2087. Le peintre verrier avait initialement prévu quatre figures symboliques.

33. - La Faculté de Pharmacie, 1882-1982. Saint-Cloud, 1982, p. 187.

34. - Arch. nat. $\mathrm{F}^{21} 4867,19$ janvier 1889, procès-verbal de la séance du Comité des Travaux d'art. Transcrit par HOTTIN, Christian. Quand la Sorbonne était peinte. Étude sur la décoration peinte et sculptée des établissements d'enseignement supérieur parisiens. Thèse de l'École des chartes, 1997, vol. VI, p. 566-568 (annexe I).

35. - COURTHION, Pierre. Georges Rouault. Suivi d'un catalogue établi avec la collaboration d'Isabelle Rouault. Paris : Flamarion, 1962, p. 26, 30. Je remercie Véronique David de m'avoir signalé cette référence.

36. - BOUYER, Raymond. « Les vitraux d'Albert Besnard ». Art et Décoration, vol. XXIX, $1^{\mathrm{er}}$ semestre 1911, p. 119-132 (p. 126).

37. - FOURCAUD, Louis de. Les arts décoratifs aux Salons. Revue des Arts décoratifs, vol. XIV, 1893-1894, p. 376-378. Art et Industrie, planches des livraisons de mai et août 1910.

38. - BOUYER, Raymond. « Les vitraux d'Albert Besnard ». Art et Décoration, vol. XXIX, $1^{\mathrm{er}}$ semestre 1911, p. 125.

39. - Arch. nat., $\mathrm{F}^{21} 2055 \mathrm{~B}$.

40. - FINANCE, Laurence de, KUKAWSKA, Katia. «Henri Carot (1850-1919), peintre verrier parisien méconnu ». Dans Un patrimoine de lumière, 1830-2000. Verrières des Hauts-de-Seine, SeineSaint-Denis, Val-de-Marne. Paris: Monum, Éditions du patrimoine, 2003, p. 297-311 (p. 309). La basse-cour intégrée au triptyque de la Vie animale réalisé en 1895 par Carot pour le député Denys Cochin est tirée d'un carton de Besnard distinct de celui de l'École de Pharmacie; voir DE FINANCE, Laurence, STAHL, Fabienne. La collaboration entre Maurice Denis et Henri Carot à la lumière de la restauration des verrières du Vésinet (Yvelines). In Situ, revue des patrimoines [en ligne], 2009, $\mathrm{n}^{\circ} 12$ [consulté le 31/10/2011].

http://www.insitu.culture.fr/article.xsp?numero=12\&id_article=financestahl-1292, note 11 et fig. 5.

41. - Salle 63, inv. DO 1981-1. Dimensions : H. 2,50 m - L. 2 m. BASCOU, Marc, MASSÉ, MarieMadeleine et THIÉBAUT, Philippe. Musée d'Orsay. Catalogue sommaire illustré des arts décoratifs. Paris, 1988.

42. - MAGNE, Lucien. «Le vitrail ». Art et Décoration, vol. I, 1897, fasc. 2, p. 16. Bibliothèque des Arts décoratifs. Album Maciet, série 482, vol. 19.

43. - HEUVRARD-BEAUVALOT, Chantal. Albert Besnard (1849-1934): une vocation de décorateur. Thèse, université de Paris X Nanterre, dir. Ségolène Le Men, 2005, p. 127, 159.

\section{RÉSUMÉS}

L'École de Pharmacie bâtie par Charles-Jean Laisné de 1877 à 1882 le long de l'avenue de l'Observatoire (Paris, $6^{\mathrm{e}}$ ) a reçu un décor soigné comprenant sculptures, peintures murales et 
vitraux. L'architecte obtint que la commande des vitraux soit adressée à Émile Hirsch, un peintre verrier parisien avec lequel il avait collaboré régulièrement sur d'autres chantiers. Hirsch livra en 1884 quatre verrières évoquant l'histoire de l'institution, mises en place dans les deux escaliers, et en 1888 un grand vitrail allégorique destiné au fond du vestibule central de l'établissement, depuis déposé. Un complément du décor vitré de cette grande salle avait été prévu pour les fenêtres latérales mais fut finalement abandonné, après que le peintre Albert Besnard, auteur des peintures murales, eût dessiné en 1889 huit vitraux à sujets animaliers. On doit cependant à des initiatives privées la réalisation par Henri Carot de deux verrières tirées des cartons de Besnard.

Built from 1877 to 1882 by Charles-Jean Laisné, the main façade of the École de Pharmacie opens onto the Avenue de l'Observatoire (Paris, 6th arr.). The new school was richly decorated with sculpture, wall-painting and stained-glass. According to the architect's wishes, Émile Hirsch, his collaborator on previous restoration operations, was commissioned for the stained-glass windows. In 1884, the parisian glazier delivered four windows with historical subjects related to the institution, which took place in the two stairways, and in 1888 an allegorical stained-glass for the main hall, now removed. Laisné intended to complete the decoration of this hall with eight new windows, representating animals in their natural surroundings. Designed in 1889 by Albert Besnard, the painter who decorated the walls of this room, they were in fact not executed. But two of them were realized later by Henri Carot for private commissioners.

INDEX

Mots-clés : Albert Besnard, Charles-Jean Laisné, école de pharmacie, Émile Hirsch, Henri Carot, verrière, vitrail

\section{AUTEUR}

\section{FRANÇOISE GATOUILLAT}

Ingénieur de recherche, Centre André Chastel, Institut national d'histoire de l'art francoise.gatouillat@paris-sorbonne.fr 